\title{
Match-making for posaconazole through systems thinking
}

\author{
Matthias A. Fügi ${ }^{1,2}$, Marcel Kaiser ${ }^{1,2}$, Marcel Tanner ${ }^{1,2}$, Roger Schneiter ${ }^{3}$, \\ Pascal Mäser ${ }^{1,2}$, and Xue Li Guan ${ }^{1,2}$
}

\author{
${ }^{1}$ Swiss Tropical and Public Health Institute, $\mathrm{CH}-4051$ Basel, Switzerland \\ ${ }^{2}$ University of Basel, $\mathrm{CH}-4000$ Basel, Switzerland \\ ${ }^{3}$ University of Fribourg, CH-1700 Fribourg, Switzerland
}

\begin{abstract}
Currently available drugs for Chagas' disease are limited by toxicity and low efficacy in the chronic stage. Posaconazole, the most advanced new anti-chagasic drug candidate, did not fully confirm its initial potential in a Phase II clinical trial for chronic Chagas' disease. Given that posaconazole is highly active against Trypanosoma cruzi in vitro, and was very well tolerated in clinical trials, it should not be abandoned. Rather, a combination therapy may provide a highly promising outlook. Systems-scale approaches facilitate the hunt for a combination partner for posaconazole, which acts by blocking sterol biosynthesis. Mounting evidence suggests the functional interactions between sterols and sphingolipids in vivo. Here, we propose combining sterol and sphingolipid biosynthesis inhibitors to advance drug development in Chagas' disease.
\end{abstract}

Chagas' disease: a global burden and the unmet need
for new drugs
Worldwide, an estimated 7 million- 8 million people are
infected with the protozoan parasite Trypanosoma cruzi
[1]. Chagas' disease is endemic in 21 South American
countries but, as a result of population mobility, also occurs
outside the continent [2]. Chagas' disease poses a global
challenge due to the lack of safe and effective treatment.
Efforts towards the urgently needed new drugs have cul-
minated in the clinical development of triazolic antifungals
for Chagas' disease. The most advanced drug candidates
were posaconazole and E1224, a prodrug of ravuconazole.
Unfortunately, although very well tolerated, both com-
pounds failed to meet the high expectations in recent
clinical Phase II trials: they did not cure chronic Chagas'
disease as indicated by the high relapse rates observed
during follow-up. Considering that posaconazole and
E1224 are highly active against $T$. cruzi and well tolerated
in clinical trials, we propose not to abandon the triazoles
but to find a suitable partner for combination therapy.
Combination therapy is an attractive approach because it
may improve treatment efficacy while decreasing the

Corresponding author: Guan, X.L. (xueli.guan@unibas.ch).

Keywords: Chagas' disease; combination therapy; systems biology; sterols; sphingolipids. likelihood of resistance development [3]. Systems biology aims at revealing interconnections of biological networks and these works serve as useful resources for rational identification of potential interacting partners for chemotherapy. Based on genetic, physical, and functional interactions between sterols and sphingolipids [4] and due to the synthetic lethality of Saccharomyces cerevisiae double mutants of sterol and sphingolipid anabolism [5], our opinion is that inhibitors of sphingolipid biosynthesis are promising combination partners for posaconazole or ravuconazole.

\section{Current drugs for the treatment of Chagas' disease}

Chagas' disease remained without an effective treatment for several decades after its original description in 1909 [6]. Nifurtimox and benznidazole, discovered over 40 years ago and still the only available drugs for the specific treatment of Chagas' disease, are limited by toxicity and low efficacy in the established chronic form of the disease [7]. These major drawbacks, along with upcoming reports of resistant T. cruzi [8] and the spread of the disease to nonendemic countries [2], spurred renewed drug research and development (R\&D) for Chagas' disease. The triazoles posaconazole and E1224 were the only candidates to pass the preclinical phase and enter clinical proof-of-concept trials. However, the results in Phase II clinical trials were disappointing. While the parasitemia dropped below detection limit after treatment, 10 months later, most patients again tested positive for T. cruzi [9]. Either candidate was less efficacious than benznidazole. This outcome is arguably attributable to limited systemic exposure resulting from the liquid suspension of the drug and suboptimal treatment duration [10]. Even so, these results have aggravated the situation in the already slim Chagas' portfolio, where the most advanced alternatives to the triazoles have not yet reached clinical Phase I.

\section{Quo vadis posaconazole?}

In 1995, V.M. Girijavallabhan described posaconazole (SCH 56592) as a novel, orally active, broad-spectrum antifungal agent [11]. Posaconazole (Nofaxil) was developed by Schering-Plough and was approved by the US Food and Drug Administration (FDA) for the treatment of invasive fungal infection in humans in 2006 [12]. Similar to other triazoles, posaconazole is a potent inhibitor of the 
Cyp450-dependent lanosterol 14 $\alpha$-demethylase (Cyp51) in yeasts and molds [13]. Inhibition of Cyp51 blocks the synthesis of ergosterol, which is an essential component in the cell membrane of fungal pathogens. Accumulation of methylated sterol precursors and disruption of the close packing of acyl chains of phospholipids in ergosterol-depleted cell membranes ultimately leads to growth inhibition of the fungi [14]. Similar to fungi, T. cruzi synthesizes ergosterol and is sensitive to sterol biosynthesis inhibitors [15]. Posaconazole exhibited excellent in vitro and in vivo efficacy against both drug-sensitive and -resistant isolates $[16,17]$. Suitable combination partners for posaconazole might be found in the sterol biosynthesis pathway to enhance the blockade of this highly interconnected metabolic network. The links between distinct steps of the sterol biosynthesis pathway can be exemplified in $S$. cerevisiae: in the presence of erg 6 deletion, the erg 2 gene product works inefficiently [4], resulting in an erg 6 single deletion mutant exhibiting a partial phenotype of an erg2erg6 double mutant. Furthermore, cells have evolved compensatory mechanisms within metabolic pathways such that accumulating substrates resulting from inhibition of a specific enzymatic step can be alternatively metabolized as a salvage mechanism. Clearly, sterol biosynthesis is of proven druggability and targeting multiple steps in the same pathway can potentiate antiparasitic activity. Lovastatin, a blockbuster used for hypercholesterolemia, enhanced the antiproliferative effects of ketoconazole and terbinafine against T. cruzi in vitro and in vivo [18]. Evidence from yeast points in the same direction because at least a dozen proteins interacting with Erg11 (Cyp51 ortholog in S. cerevisiae) can be found in the sterol metabolic pathway [19]. Druggable pathways that interact with sterol metabolism also represent complementary targets. Glycerophospholipid biosynthesis inhibitors, such as ajoene or alkyl-lysophospholipids (ALP, e.g., miltefosine), have been shown to have antiproliferative effects on $T$. cruzi epimastigotes and amastigotes [20-22]. Growth inhibition correlated with a decrease in the phosphatidylcholine to phosphatidylethanolamine ratio (PC:PE) and, in the case of ALP, also with a marked effect on sterol composition due to inhibition of sterol 22desaturase (Erg5), a finding that probably explains the antiproliferative synergism of these drugs with the Cyp51 inhibitor ketoconazole against both proliferative stages (epimastigotes and intracellular amastigotes) of the parasite [21,22]. Here, we propose to combine the anti-chagasic triazoles with inhibitors of sphingolipid synthesis, as suggested by systems approaches.

\section{Systems-based matchmaking}

Systems approaches were pioneered in model organisms to understand how biological systems act as a whole. Emergence of complex behavior is observed when the biological systems are treated as networks. These can be protein interaction networks, metabolic networks, or genetic networks. All are amenable to large-scale interaction studies, particularly in S. cerevisiae, where global approaches such as chemical genetics screens, mutant library screens, protein-protein interactions, and other -omics technologies can be automated. These have led to the availability of databases containing a wealth of information that can be mined to generate new hypotheses of cellular and system functions. It can also guide drug discovery in modern medicine by providing a rational basis to pinpoint interrelated pathways. The interacting partners for CYP51 in $S$. cerevisiae, for instance, are found on the BioGRID database [23], containing 103 physical and 184 genetic interactions. Candidate pathways can be further narrowed by phenotypes and functionality. Specifically, interactors of Cyp51 that cause synthetic lethality will be appealing.

\section{Capitalizing on sterol-sphingolipid interactions as a combinatorial treatment}

Posaconazole blocks sterol biosynthesis; thus, druggable pathways interacting with sterol metabolism and functions represent highly complementary matches for posaconazole. Sterols have been shown to modulate membrane thickness in artificial membranes and this property has been proposed to have a role in membrane protein localisation in vivo [24]. It is increasingly known that proteins and lipids do not freely diffuse over the entire surface of the cell and it has been proposed that eukaryotic plasma membranes contain micro- and/or nanodomains (reviewed in [25-27]) that act as platforms creating membrane heterogeneities with many proposed functions. There is clear biophysical evidence that sterols and sphingolipids can segregate from other lipids in simple artificial membrane systems to form liquid ordered domains [28].

Sterol-sphingolipid interactions have also been demonstrated in vivo. Evidence in the budding yeast, S. cerevisiae, suggests a genetic interaction between mutants in sterol and sphingolipid biosynthesis [4,5,29,30]. For example, mutants that affect the hydroxylation pattern of sphingolipids display synthetic growth defects with mutations in late-acting ergosterol biosynthetic genes [4]. By contrast, mutations that affect the synthesis of the sphingolipidspecific very-long chain $\mathrm{C}_{26}$ fatty acid display strong synthetic lethality with mutations in ERG6, a methyltransferase that catalyzes the addition of a fungal-specific methyl group at position $\mathrm{C}_{24}$ in the aliphatic side chain or ergosterol [5]. Figure 1 summarizes experimental evidence on genetic interactions between the sterol and sphingolipid synthetic genes. While the bulk of experimental evidence comes from high throughput screens and needs to be treated with caution, there is solid support for synthetic lethality between ELO3 and ERG6 [5], and for synthetic growth defects of sterol synthetic genes with ISC1, SUR2, and SCS7 [4]. Strikingly, synthetic lethality has been demonstrated between CYP51 and SCS7 [31].

In yeast and higher eukaryotes, it has further been shown that sterols and sphingolipids are important for proper trafficking of transporters (amino acids and proton pumps) to the cell surface and their stability at the plasma membrane [32-35]. Adaptation to changes in sterol composition by adjusting sphingolipid levels and variants is not unique to the unicellular eukaryotes but is also present in Metazoa, exemplified by the fruit fly, Drosophila melanogaster. As a sterol auxotroph, D. melanogaster cannot synthesize sterols but this lipid is required for larval growth and development. A drop in sterol levels caused developmental arrest but cells remain viable, possibly due to a compensatory increase in sphingolipid levels and composition [36]. 


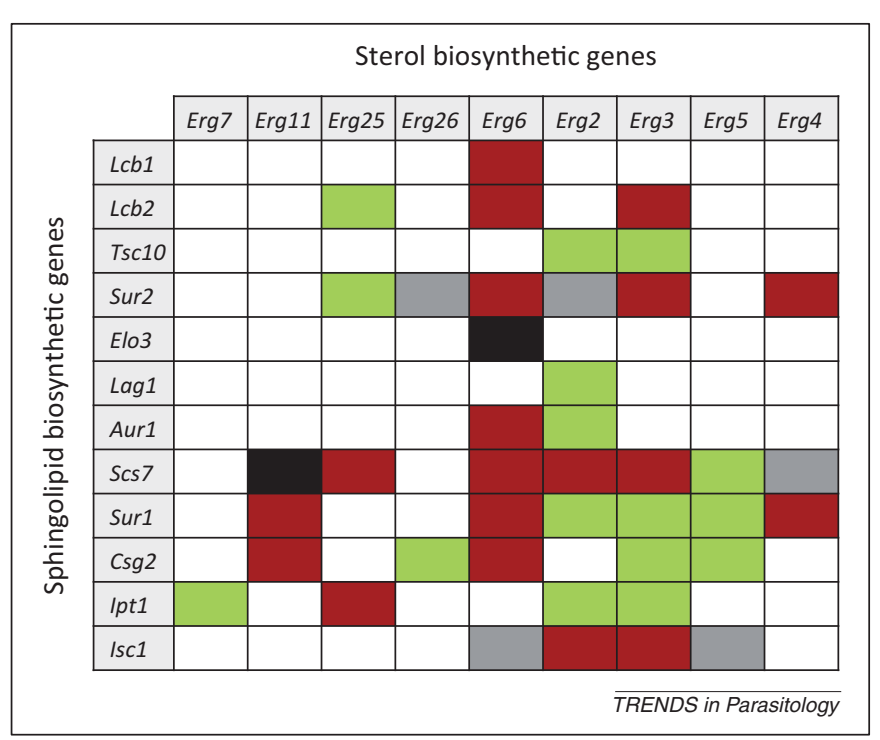

Figure 1. Genetic interactions between sterol and sphingolipid biosynthetic genes in yeast. Only genes for which an interaction has been experimentally documented are shown. Data are from BioGRID v. 3.2.117 [23]. Green, positive genetic interaction; red, negative genetic interaction or synthetic growth defect; black, synthetic lethality; gray, equivocal results from different screens. For gene symbol definitions, please see Figure 2.

Figure 2 shows the structures of mannosyl diinositol phosphoryl ceramide $\left[\mathrm{M}(\mathrm{IP})_{2} \mathrm{C}\right]$ and ergosterol, which is the most abundant sphingolipid and sterol species in yeast. Together with glycerophospholipids, sterols and sphingolipids comprise the major classes of eukaryotic membrane lipids. Many membrane characteristics, such as composition and integrity, turnover or trafficking, and signaling, fulfill the requirements for bona fide drug targets in parasites: they must be (i) essential for parasite survival; (ii) druggable; and (iii) sufficiently different from the host. Indeed, 'membrane-lipid therapy' was coined by Pablo Escribá and is defined as the therapeutic approach based on the regulation of the membrane-lipid composition and structure to modulate cell functions [37]. In a broader sense, we think of membrane therapy as interfering with membranes directly or via curtailing lipid biosynthesis.

While there currently is no evidence in $T$. cruzi on the interactions of sterols and sphingolipids, it is intuitive that the simultaneous inhibition of both sterol and sphingolipid metabolism will have a major impact on membrane homeostasis. Moreover, there are several lines of evidence that these lipids have critical roles in trypanosomatids. Endogenous sterols and sphingolipids are required for proliferation of trypanosomes [38-40]. Interestingly, reduced inositolphosphoceramide (IPC) levels due to inhibition of serine palmitoyltransferase (Spt2) in T. brucei have been shown to be compensated for by increased levels of phosphatidylcholine and cholesterol, demonstrating a tight interaction of sterol and sphingolipid homeostasis [41]. As in yeast, IPC rather than glycerophospholipids is utilized as lipid anchor constituent of glycoproteins and free glycosylinositolphospholipids (GIPLs) in T. cruzi [42]. Furthermore, inhibition of IPC synthesis impaired T. cruzi differentiation [43].

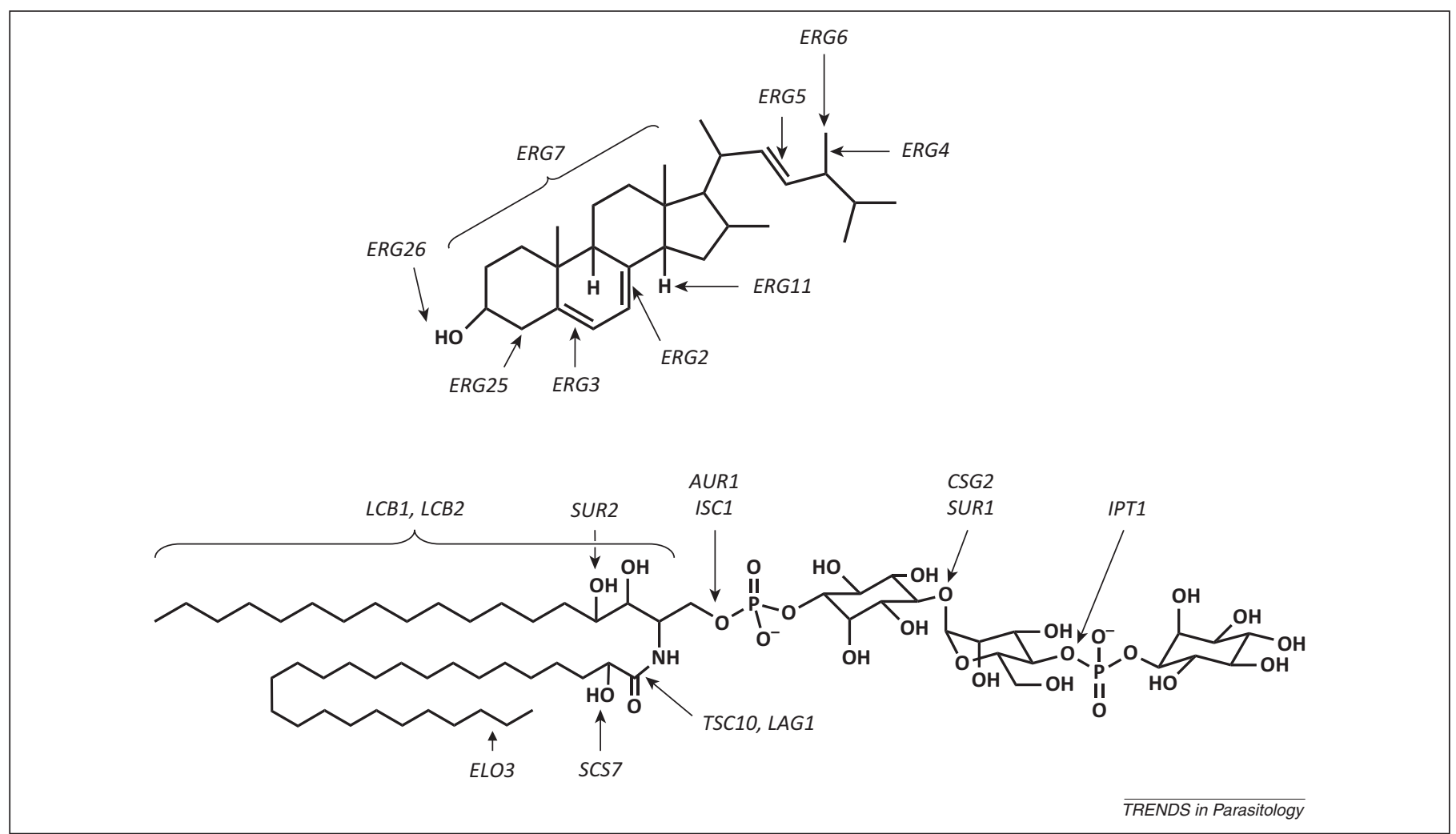

Figure 2. Structures of ergosterol and the sphingolipid, mannosyl diinositol phosphoryl ceramide $\left[\mathrm{M}(\mathrm{IP})_{2} \mathrm{C}\right]$. Genes shown encode enzymes that catalyze major steps in sterol and sphingolipid metabolism, respectively. The list of genes is not exhaustive but based on sterol/sphingolipid interactions (see also Figure 1). For ergosterol: $E R G 7$, 2,3-oxidosqualene cyclase; ERG11, $\mathrm{C}_{14}$ demethylation; ERG25, $\mathrm{C}_{4 \alpha}$ methyl oxidation; ERG26, $\mathrm{C}_{3}$ decarboxylation; ERG6, $\mathrm{C}_{24}$ methylation; ERG2, $\mathrm{C}_{8}$ isomerization; ERG3, $\mathrm{C}_{5}$ desaturation; ERG5, $\mathrm{C}_{22}$ desaturation; ERG4, $\mathrm{C}_{24}$ reduction. For $\mathrm{M}(\mathrm{IP})_{2} \mathrm{C}$ : LCB1, serine C-palmitoyltransferase; LCB2, serine C-palmitoyltransferase; TSC10, 3dehydrosphinganine reductase; SUR2, long chain base hydroxylase; ELO3, fatty acyl elongase; LAG1, sphingosine N-acyltransferase; AUR1, phosphatidylinositol:ceramide phosphoinositol transferase; SCS7, fatty acyl hydroxylase; SUR1, mannosylinositol phosphorylceramide (MIPC) synthase; CSG2, MIPC synthase regulatory subunit; IPT1, inositolphosphotransferase; ISC1, inositol phosphosphingolipid phospholipase C. 
Table 1. Sphingolipid biosynthesis inhibitors in clinical trials or on the market

\begin{tabular}{|c|c|c|c|c|}
\hline Compound name & $\begin{array}{l}\text { Clinical phase or drug } \\
\text { name (if on the market) }\end{array}$ & Mechanism of action & Indication & Refs \\
\hline $\mathrm{N}$-butyldeoxynojirimycin & Miglustat, Zavesca $\AA$ & Glucosylceramide synthase inhibitor & Gaucher disease & \\
\hline FTY720 & Gilenya & Sphingosine-1-phosphate receptor inhibitor & Multiple sclerosis & [49] \\
\hline Safingol & Phase I & Sphingosine kinase inhibitor & Cancer & [50] \\
\hline Phenoxodiol & Phase III & Sphingosine kinase inhibitor & Cancer & [51] \\
\hline ABC294640 & Phase I & Sphingosine kinase inhibitor & Cancer & [52] \\
\hline Sphingomab & Preclinical & Anti-sphingosine-1-phosphate antibody & Cancer & [53] \\
\hline Fenretinide & Phase I & Ceramide desaturase inhibitor & Cancer & [54] \\
\hline Desipramine & $\begin{array}{l}\text { Treyzafagit, Norpramin, } \\
\text { and Pertofrane }\end{array}$ & Acid sphingomyelinase inhibitor & Antidepressant & \\
\hline Imiglucerase & Cerezyme & $\beta$-glucocerebrosidase replacement & Gaucher disease & \\
\hline \multirow[t]{3}{*}{ Amitriptyline } & Phase IIb & Acid sphingomyelinase inhibitor & Cystic Fibrosis & [55] \\
\hline & \multirow[t]{2}{*}{ Elavil, Endep, and Vanatrip } & & Antidepressant & \\
\hline & & & Analgesic & \\
\hline Fluoxetine & $\begin{array}{l}\text { ROzac, PROzac Weekly, } \\
\text { Sarafem, Rapiflux, Selfemra, } \\
\text { and PROzac Pulvules }\end{array}$ & Acid sphingomyelinase inhibitor & Antidepressant & \\
\hline Aureobasidin $\mathrm{A}^{\mathrm{a}}$ & Phase I & Inositol phosphorylceramide synthase inhibitor & Antifungal & [56] \\
\hline
\end{tabular}

The relation between sterols and sphingolipids, evident in yeast and the fruit fly, could indicate a potential evolutionarily conserved adaption mechanism for membrane homeostasis. Thus, concomitant perturbation of these two classes of lipids may promote synergistic lethality. Therefore, it will be interesting to test the interactions between posaconazole or ravuconazole and sphingolipid inhibitors on $T$. cruzi focusing on $100 \%$ cidality rather than potential synergism.

\section{Concluding remarks and outstanding questions}

Sterile cidality also against nonproliferating trypanosomes is imperative to Chagas' disease chemotherapy. To this aim, three different strategies have been proposed to select a suitable combination partner for azoles. The partner could be a drug such as benznidazole, which is $100 \%$ cidal itself and additive in action with posaconazole [44-46]. It could also be a drug that is not $100 \%$ cidal itself but shows synergistic interaction with posaconazole, such as amiodarone, amlodipine, or clemastine [46,47]. In addition, aiming to completely block sterol synthesis, the combination partner could be another sterol biosynthesis inhibitor $[15,18]$. Here, we propose as an additional strategy the partnership between posaconazole and sphingolipid inhibitors. This is based on the hypothesis that such a combination will be most effective in disrupting membrane integrity and functions, which is critical also for quiescent cells. Exploration of the chemotherapeutic potential of this proposed partnership will require: (i) systems knowledge of T. cruzi lipid physiology; (ii) sphingolipid biosynthesis inhibitors; and (iii) a test for $100 \%$ sterile cidality on the relevant $T$. cruzi stages.

Currently, there is no evidence that sterols and sphingolipids functionally interact in $T$. cruzi. The advancing technologies for system-scale analyses of genes, transcripts, proteins, and metabolites (including lipids) accompanied by high throughput genetic and chemical screening, will revolutionize our understanding of $T$. cruzi biology and identify possible pathways for combination therapies. Concomitant chemotherapeutic attack of sterol

Table 2. Sterol biosynthesis inhibitors ${ }^{a}$

\begin{tabular}{|c|c|c|c|}
\hline Class & Target and/or mechanism of action & Indication & Refs \\
\hline Statins & $\begin{array}{l}\text { Competitive inhibitors of HMG-CoA reductase, preventing the formation of } \\
\text { mevalonate from HMG-CoA; they occupy the HMG-binding pocket and part of } \\
\text { the binding surface for CoA }\end{array}$ & $\begin{array}{l}\text { Used as cholesterol- } \\
\text { lowering drugs in humans }\end{array}$ & {$[57,58]$} \\
\hline Bisphosphonates (BPs) & $\begin{array}{l}\text { Potent inhibitors of bone resorption. The selective action on bone is based on } \\
\text { the binding of the BP moiety to the bone mineral; nitrogen-containing BPs bind } \\
\text { to, and inhibit the activity of, farnesyl diphosphate synthase }\end{array}$ & $\begin{array}{l}\text { Used to treat osteoporosis } \\
\text { and other bone resorption } \\
\text { diseases }\end{array}$ & [59-61] \\
\hline $\begin{array}{l}\text { Quinuclidines and/ } \\
\text { or zaragozic acids }\end{array}$ & $\begin{array}{l}\text { Inhibition of squalene synthase (SOS); quinuclidines may inhibit SOS by } \\
\text { acting as carbocation mimics for FPP to squalene conversion. The aryl units } \\
\text { may act as isosteres for the isoprenyl subunits in the farnesyl chain. }\end{array}$ & Not in clinical use & [62] \\
\hline Allylamines & Specific inhibition of fungal squalene mono-oxygenase & $\begin{array}{l}\text { Used for topical treatment } \\
\text { of fungal infections }\end{array}$ & {$[63,64]$} \\
\hline Azoles & $\begin{array}{l}\text { Bind as the sixth ligand to the haem in lanosterol } 14 \alpha \text {-demethylase (=CYP51), } \\
\text { thus occupying the active site and acting as noncompetitive inhibitors; } \\
\text { blocking the synthesis of ergosterol leads to the accumulation of methylated } \\
\text { sterol precursors }\end{array}$ & $\begin{array}{l}\text { Used to treat fungal } \\
\text { infections }\end{array}$ & {$[65,66]$} \\
\hline Azasterols & $\begin{array}{l}\text { Evidence from yeast shows that azasterols inhibit the enzyme C24-sterol } \\
\text { methyltransferase }\end{array}$ & Not in clinical use & [67] \\
\hline
\end{tabular}


and sphingolipid biosynthesis is facilitated by the availability of sphingolipid inhibitors [48], owing to the interests in their functions in human health. As with every drug candidate, consideration must be given to the potential toxicity of sphingolipid biosynthesis inhibitors. Toxicity is one reason why most of the numerous existing sphingolipid inhibitors remain experimental compounds [48]. Nonetheless, this class of compounds is promising, because there are several in clinical use or in clinical trials for a spectrum of human diseases (Table 1) and, given the role of sphingolipids in many other human diseases, efforts to discover novel compounds are ongoing. The same applies for sterol biosynthesis inhibitors (Table 2). A crucial requirement for R\&D of next-generation anti-chagasic agents will be an in vitro test that is amenable to medium throughput and that can demonstrate $100 \%$ cidality against nonproliferating intracellular amastigote $T$. cruzi. Such an assay must be able to predict the lack of sterile cidality of posaconazole and ravuconazole.

In summary, we argue that the potential of posaconazole must be further explored with a view of rational target identification and achieving combination therapy through systems-scale approaches. Based on evidence in model organisms, particularly the budding yeast, the matching of sphingolipid synthesis inhibitors as partners of triazoles can impair membrane functionality and, thus, may kill proliferating as well as dormant parasites.

\section{Acknowledgments}

X.L.G. was supported by the Swiss National Science Foundation Ambizione (PZ00P3_136738). The authors thank the Drugs for Neglected Diseases initiative (DNDi) and Professor Howard Riezman (University of Geneva) for helpful discussions.

\section{References}

1 Crompton, D.W. (ed.) (2013) Sustaining the Drive to Overcome the Global Impact of Neglected Tropical Diseases, World Health Organization

2 Coura, J.R. and Viñas, P.A. (2010) Chagas disease: a new worldwide challenge. Nature 465, S6-S7

3 Ehrlich, P. (1907) Chemotherapeutische Trypanosomen-Studien, Berliner klinische Wochenschrift

4 Guan, X.L. et al. (2009) Functional interactions between sphingolipids and sterols in biological membranes regulating cell physiology. Mol. Biol. Cell 20, 2083-2095

5 Eisenkolb, M. et al. (2002) A specific structural requirement for ergosterol in long-chain fatty acid synthesis mutants important for maintaining raft domains in yeast. Mol. Biol. Cell 13, 4414-4428

6 Chagas, C. (1909) Nova tripanozomiaze humana: estudos sobre a morfolojia e o ciclo evolutivo do Schizotrypanum cruzi n. gen., n. sp., ajente etiolojico de nova entidade morbida do homem. Mem. Inst. Oswaldo Cruz 1, 159-218

7 Urbina, J.A. (2010) Specific chemotherapy of Chagas disease: relevance, current limitations and new approaches. Acta Trop. 115, $55-68$

8 Filardi, L.S. and Brener, Z. (1987) Susceptibility and natural resistance of Trypanosoma cruzi strains to drugs used clinically in Chagas disease. Trans. R. Soc. Trop. Med. Hyg. 81, 755-759

9 Molina, I. et al. (2014) Randomized trial of posaconazole and benznidazole for chronic Chagas' disease. N. Engl. J. Med. 370, 1899-1908

10 Urbina, J.A. (2014) Recent clinical trials for the etiological treatment of chronic Chagas disease: advances, challenges and perspectives. $J$. Eukaryot. Microbiol. Published online October 5, 2014, (http:// dx.doi.org/10.1111/jeu.12184)
11 Girijavallabhan, V.M. et al. (1995) Sch 56592, a novel orally active broad spectrum antifungal agent. Abstr. Intersci. Conf. Antimicrob. Agents Chemother. 35, 123

12 Schering-Plough (2006) Kenilworth Schering-Plough Announces FDA Approval of Noxafil for Treatment of Oropharyngeal Candidiasis, MedNews

13 Munayyer, H.K. et al. (2004) Posaconazole is a potent inhibitor of sterol 14alpha-demethylation in yeasts and molds. Antimicrob. Agents Chemother. 48, 3690-3696

14 Sheehan, D.J. et al. (1999) Current and emerging azole antifungal agents. Clin. Microbiol. Rev. 12, 40-79

15 Maldonado, R.A. et al. (1993) Experimental chemotherapy with combinations of ergosterol biosynthesis inhibitors in murine models of Chagas' disease. Antimicrob. Agents Chemother. 37, 1353-1359

16 Urbina, J.A. et al. (1998) Antiproliferative effects and mechanism of action of SCH 56592 against Trypanosoma (Schizotrypanum) cruzi: in vitro and in vivo studies. Antimicrob. Agents Chemother. 42, 1771-1777

17 Molina, J. et al. (2000) Activities of the triazole derivative SCH 56592 (posaconazole) against drug-resistant strains of the protozoan parasite Trypanosoma (Schizotrypanum) cruzi in immunocompetent and immunosuppressed murine hosts. Antimicrob. Agents Chemother. 44, 150-155

18 Urbina, J.A. et al. (1993) Mevinolin (lovastatin) potentiates the antiproliferative effects of ketoconazole and terbinafine against Trypanosoma (Schizotrypanum) cruzi: in vitro and in vivo studies. Antimicrob. Agents Chemother. 37, 580-591

19 Mo, C. and Bard, M. (2005) A systematic study of yeast sterol biosynthetic protein-protein interactions using the split-ubiquitin system. Biochim. Biophys. Acta 1737, 152-160

20 Urbina, J.A. et al. (1993) Inhibition of phosphatidylcholine biosynthesis and cell proliferation in Trypanosoma cruzi by ajoene, an antiplatelet compound isolated from garlic. Biochem. Pharmacol. 45, 2381-2387

21 Lira, R. et al. (2001) Mechanism of action of anti-proliferative lysophospholipid analogues against the protozoan parasite Trypanosoma cruzi: potentiation of in vitro activity by the sterol biosynthesis inhibitor ketoconazole. J. Antimicrob. Chemother. 47, 537-546

22 Santa-Rita, R.M. et al. (2005) Anti-proliferative synergy of lysophospholipid analogues and ketoconazole against Trypanosoma cruzi (Kinetoplastida: Trypanosomatidae): cellular and ultrastructural analysis. J. Antimicrob. Chemother. 55, 780-784

23 Stark, C. et al. (2006) BioGRID: a general repository for interaction datasets. Nucleic Acids Res. 34, D535-D539

24 Bretscher, M.S. and Munro, S. (1993) Cholesterol and the Golgi apparatus. Science 261, 1280-1281

25 Simons, K. and Ikonen, E. (1997) Functional rafts in cell membranes. Nature 387, 569-572

26 Jacobson, K. et al. (2007) Lipid rafts: at a crossroad between cell biology and physics. Nat. Cell Biol. 9, 7-14

27 Kusumi, A. et al. (2005) Paradigm shift of the plasma membrane concept from the two-dimensional continuum fluid to the partitioned fluid: high-speed single-molecule tracking of membrane molecules. Annu. Rev. Biophys. Biomol. Struct. 34, 351-378

28 Ahmed, S.N. et al. (1997) On the origin of sphingolipid/cholesterol-rich detergent-insoluble cell membranes: physiological concentrations of cholesterol and sphingolipid induce formation of a detergent-insoluble, liquid-ordered lipid phase in model membranes. Biochemistry 36, 10944-10953

29 Baudry, K. et al. (2001) The effect of the erg26-1 mutation on the regulation of lipid metabolism in Saccharomyces cerevisiae. J. Biol. Chem. 276, 12702-12711

30 Jin, H. et al. (2008) Ergosterol promotes pheromone signaling and plasma membrane fusion in mating yeast. J. Cell Biol. 180, 813-826

31 Parsons, A.B. et al. (2004) Integration of chemical-genetic and genetic interaction data links bioactive compounds to cellular target pathways. Nat. Biotechnol. 22, 62-69

32 Umebayashi, K. and Nakano, A. (2003) Ergosterol is required for targeting of tryptophan permease to the yeast plasma membrane. $J$. Cell Biol. 161, 1117-1131

33 Chung, N. et al. (2000) Sphingolipids signal heat stress-induced ubiquitin-dependent proteolysis. J. Biol. Chem. 275, 17229-17232 
34 Proszynski, T.J.et al. (2005) A genome-wide visual screen reveals a role for sphingolipids and ergosterol in cell surface delivery in yeast. Proc. Natl. Acad. Sci. U.S.A. 102, 17981-17986

35 Gaigg, B. et al. (2006) Very long-chain fatty acid-containing lipids rather than sphingolipids per se are required for raft association and stable surface transport of newly synthesized plasma membrane ATPase in yeast. J. Biol. Chem. 281, 34135-34145

36 Carvalho, M. et al. (2010) Survival strategies of a sterol auxotroph. Development 137, 3675-3685

37 Escribá, P.V. (2006) Membrane-lipid therapy: a new approach in molecular medicine. Trends Mol. Med. 12, 34-43

38 Urbina, J.A. (2002) Chemotherapy of Chagas disease. Curr. Pharm. Des. 8, 287-295

39 Urbina, J.A. and Docampo, R. (2003) Specific chemotherapy of Chagas disease: controversies and advances. Trends Parasitol. 19, 495-501

40 Sutterwala, S.S. et al. (2007) De novo sphingolipid synthesis is essential for viability, but not for transport of glycosylphosphatidylinositolanchored proteins, in African trypanosomes. Eukaryot. Cell 6, 454-464

41 Fridberg, A. et al. (2008) Sphingolipid synthesis is necessary for kinetoplast segregation and cytokinesis in Trypanosoma brucei. J. Cell Sci. 121, 522-535

42 De Lederkremer, R.M. and Agusti, R. (2009) Glycobiology of Trypanosoma cruzi. Adv. Carbohydr. Chem. Biochem. 62, 311-366

43 Salto, M.L. et al. (2003) Formation and remodeling of inositolphosphoceramide during differentiation of Trypanosoma cruzi from trypomastigote to amastigote. Eukaryot. Cell 2, 756-768

44 Cencig, S. et al. (2012) Evaluation of benznidazole treatment combined with nifurtimox, posaconazole or AmBisome $\mathbb{R}$ in mice infected with Trypanosoma cruzi strains. Int. J. Antimicrob. Agents 40, 527-532

45 Diniz, L. and de, F. et al. (2013) Benznidazole and posaconazole in experimental Chagas disease: positive interaction in concomitant and sequential treatments. PLoS Negl. Trop. Dis. 7, e2367

46 Planer, J.D. et al. (2014) Synergy testing of FDA-approved drugs identifies potent drug combinations against Trypanosoma cruzi. PLoS Negl. Trop. Dis. 8, e2977

47 Benaim, G. et al. (2006) Amiodarone has intrinsic anti-Trypanosoma cruzi activity and acts synergistically with posaconazole. J. Med. Chem. 49, 892-899

48 Delgado, A. et al. (2006) Inhibitors of sphingolipid metabolism enzymes. Biochim. Biophys. Acta 1758, 1957-1977

49 Brinkmann, V. et al. (2010) Fingolimod (FTY720): discovery and development of an oral drug to treat multiple sclerosis. Nat. Rev. Drug Discov. 9, 883-897

50 Dickson, M.A. et al. (2011) A phase I clinical trial of safingol in combination with cisplatin in advanced solid tumors. Clin. Cancer Res. 17, 2484-2492

51 Fotopoulou, C. et al. (2014) Weekly AUC2 carboplatin in acquired platinum-resistant ovarian cancer with or without oral phenoxodiol, a sensitizer of platinum cytotoxicity: the phase III OVATURE multicenter randomized study. Ann. Oncol. 25, 160-165
52 Kummetha Venkata, J. et al. (2014) Inhibition of sphingosine kinase 2 down-regulates the expression of $\mathrm{c}-\mathrm{Myc}$ and Mcl-1 and induces apoptosis in multiple myeloma. Blood Published online August 14, 2014, (http://dx.doi.org/10.1182/blood-2014-03-559385)

53 Sabbadini, R.A. (2011) Sphingosine-1-phosphate antibodies as potential agents in the treatment of cancer and age-related macular degeneration. Br. J. Pharmacol. 162, 1225-1238

54 Maurer, B.J. et al. (2013) Phase I trial of fenretinide delivered orally in a novel organized lipid complex in patients with relapsed/refractory neuroblastoma: a report from the New Approaches to Neuroblastoma Therapy (NANT) consortium. Pediatr. Blood Cancer 60, 1801-1808

55 Nährlich, L. et al. (2013) Therapy of CF-patients with amitriptyline and placebo: a randomised, double-blind, placebo-controlled phase IIb multicenter, cohort-study. Cell. Physiol. Biochem. 31, 505-512

56 Takesako, K. et al. (1993) Biological properties of aureobasidin A, a cyclic depsipeptide antifungal antibiotic. J. Antibiot. (Tokyo) 46, 1414 1420

57 Endo, A. et al. (1976) Competitive inhibition of 3-hydroxy-3methylglutaryl coenzyme A reductase by ML-236A and ML-236B fungal metabolites, having hypocholesterolemic activity. FEBS Lett. $72,323-326$

58 Istvan, E.S. and Deisenhofer, J. (2001) Structural mechanism for statin inhibition of HMG-CoA reductase. Science 292, 1160-1164

59 Rodan, G.A. (1998) Mechanisms of action of bisphosphonates. Annu. Rev. Pharmacol. Toxicol. 38, 375-388

60 Dunford, J.E. et al. (2001) Structure-activity relationships for inhibition of farnesyl diphosphate synthase in vitro and inhibition of bone resorption in vivo by nitrogen-containing bisphosphonates. $J$. Pharmacol. Exp. Ther. 296, 235-242

61 Kavanagh, K.L. et al. (2006) The molecular mechanism of nitrogencontaining bisphosphonates as antiosteoporosis drugs. Proc. Natl. Acad. Sci. U.S.A. 103, 7829-7834

62 Brown, G.R. et al. (1996) Synthesis and activity of a novel series of 3biarylquinuclidine squalene synthase inhibitors. J. Med. Chem. 39, 2971-2979

63 Ryder, N.S. (1992) Terbinafine: mode of action and properties of the squalene epoxidase inhibition. Br. J. Dermatol. 126 (Suppl 39), 2-7

64 Nowosielski, M. et al. (2011) Detailed mechanism of squalene epoxidase inhibition by terbinafine. J. Chem. Inf. Model. 51, 455-462

65 Jefcoate, C.R. (1978) Measurement of substrate and inhibitor binding to microsomal cytochrome P-450 by optical-difference spectroscopy. Methods Enzymol. 52, 258-279

66 Monk, B.C. et al. (2014) Architecture of a single membrane spanning cytochrome $\mathrm{P} 450$ suggests constraints that orient the catalytic domain relative to a bilayer. Proc. Natl. Acad. Sci. U.S.A. 111, 3865-3870

67 Pierce, H.D. et al. (1978) Azasterol inhibitors in yeast. Inhibition of the 24-methylene sterol delta24(28)-reductase and delta24-sterol methyltransferase of Saccharomyces cerevisiae by 23 -azacholesterol. Biochim. Biophys. Acta 529, 429-437 